\title{
Nottingham Prognostic Index is an Applicable Prognostic Tool in Non-Metastatic Triple-Negative Breast Cancer
}

\author{
O Al jarroudi*, A Zaimi, S A Brahmi, S Afqir
}

\begin{abstract}
Introduction: Triple-negative breast cancer (TNBC) is characterized by a poor prognosis due to high mortality and early relapse, requiring the study of its prognostic factors. Tumor size, histological grade and lymph node status represent important parameters that are widely studied in breast cancer, and are retained as prognostic factors by several international guidelines. The Nottingham team combined these parameters into a prognostic score called the Nottingham prognostic index (NPI). In this study, we investigated the influence of NPI on outcomes in non metastatic TNBC. Methodology: This retrospective cohort study included all female patients with non metastatic TNBC who received treatment at the Regional Oncology Center Hassan II Oujda - Morocco, between January 2009 and December 2011. The prognostic impact of the NPI on the survival curves at 5 years was studied using multivariate Cox proportional hazards models. Results: The analysis of the data involved 98 patients, 39 patients $(39.8 \%)$ were classed in the poor prognosis group with a NPI $>5.4$. The Overall survival (OS) and Disease free survival (DFS) rates at 5 years, in this group, were 70 and $55.6 \%$ respectively. After adjusting for clinic-pathological variables, a NPI $>5.4$ was associated with mortality (HR: $2.598,95 \% \mathrm{CI}: 1.423-4.744, \mathrm{p}=0.002$ ) and disease progression (HR: 2.512, CI to 95\%: 1.496-4.219, $\mathrm{p}<0.001)$ in patients with non-metastatic TNBC. Conclusion: This retrospective cohort analysis showed that NPI was an independent prognostic factor for OS and DFS at 5 years in women with non metastatic TNBC. Once validated, the impact of this score on survival outcomes could be considered in the clinical management of TNBC.
\end{abstract}

Keywords: Nottingham prognostic index- triple-negative- prognosis

Asian Pac J Cancer Prev, 20 (1), 59-63

\section{Introduction}

TNBC is defined as tumors that lack expression of estrogen receptor (ER), progesterone receptor (PR) and human epidermal growth factor 2 (HER2) (William, 2010). It constitutes one of five intrinsic subgroups of breast cancer identified by c DNA microarray analysis (Perou et al., 2000).

TNBC seems to disseminate to axillary nodes and bones less frequently than non TNBC, presenting a preferential hematogenous route with a proclivity to develop metastatic deposits in viscera, particularly in the lungs and brain (Hicks et al., 2006; Fulford et al., 2007), explaining its poor prognosis compared to other breast cancer subtypes (Dent et al., 2009; Liedtke et al., 2008).

A personnalised management which requires robust risk stratification based not only on outcome prediction but also on a biological basis is requested (D'Eredita et al., 2001). One of the Methods that have been developed to assist in predicting patient outcome and to support clinical decision making in breast cancer management is NPI (Galea et al., 1992). This score validated in large multicentre studies, is based on a combination of histopathological examination of tumour size, lymph node stage and tumour grading assembled in a prognostic index formula (Haybittle et al., 1982), stratifying patients into prognostic groups: Excellent, Good, Moderate and Poor (Balmey et al., 2007)

As above mentioned, TNBCs are believed to infrequently disseminate to axillary lymph nodes in favour of distant and visceral metastatic spread, an assumption that theoretically would impair the reliability of using NPI as a tool for TNBC prognostication (Jeanny et al., 2017; Albergaria et al., 2011). Therefore, the aim of our study is to evaluate the prognostic influence of NPI in TNBC.

\section{Materials and Methods}

\section{Patients and Methods}

We performed a retrospective study to evaluate the prognostic value of NPI in 98 patients with TNBC who received treatment for early stage breast cancer at the Regional Oncology Center Hassan II-Oujda, Morocco, between January 2009 and December 2011. Exclusion criteria included ER or PR positivity, HER2 overexpression/ amplification, and distant metastasis at 
the initial diagnosis.

All patients at risk for relapse received adjuvant chemotherapy using sequential strategy of anthracycline and taxane regimens, followed by radiotherapy if indicated.

The triple-negative tumors in our study were defined by an association of RE at $0 \%, \mathrm{RP}$ at $0 \%$, and HER-2 not overexpressed, a score of HER-2 at 0,1 or 2 with CISH or FISH negative.

Information was available for all patients' tumour size, number of positive lymph nodes, histological grade. NPI was calculated for each of the patients by using the following equation: NPI $=0.2 \times$ tumour size $(\mathrm{cm})+$ grade (1-3) + lymph node status (1-3) (Fong et al., 2015). Patients were grouped into four categories according to the NPI score: I (excellent) $\leq 2.4$; II (good) $>2.4$ but $\leq 3.4$; III (moderate) $>3.4$ but $\leq 5.4$; and IV (poor) $>5.4$. We identified 5.4 as the cut-off points of NPI.

In this study, we considered OS and DFS at 5 years as end-points. OS was defined as the length of time from the date of diagnosis until either the date of death (from any cause) or the date of last follow-up. DFS was determined as the length of time from the date of diagnosis of this disease to the date of the first signs of progress confirmed by the investigator in the medical record, or the date of death or date of latest news when the patient is censored.

The statistical analysis was performed by SPSS 21.0 software. Associations between NPI and other characteristics were analyzed using the chi-square test. For the assessment of the influence of NPI on survival outcome (OS and DFS at 5 years), we used the KaplanMeier method at univariate analysis and Cox regression models were fitted to estimate hazard ratios [HR, 95\% confidence interval $(\mathrm{CI})$ ] for the NPI. For all analysis, a significant level of $5 \%$ was considered.

\section{Results}

\section{Characteristics of patients}

Using the data from 98 non metastatic TNBC patients treated in our center, we explored each clinical pathological feature that constitutes the NPI equation. The mean tumour size was $3 \mathrm{~cm} \pm 1.2$. Concerning histological grade, $57.1 \%$ were grade III Scarff-Bloom-Richardson. $48.9 \%$ of our patients had positive lymph nodes.

We calculated NPI in all patients. This score ranged from 3-7 (Mean 4.75). It stratified our patients into Good (5.1\%), Moderate (55.1\%) and poor prognosis (39.8\%). None of the cases was scored as NPI $<3.4$ (classed in Excellent prognosis group), reinforcing the poor prognosis of this breast cancer subtype (Table 1).

Using Chi-square test, we evaluated the association of each of Nottingham prognostic components to the NPI augmentation, significant association of tumour size $(p=0.049)$, histological grade $(p=0.05)$ and lymph node status $(\mathrm{p}<0.001)$ to high scores of NPI $(>5.4)$ in TNBC (Table 2).

Tumour size is theoretically associated with the increased likelihood of lymph node invasion in breast cancer. An interesting finding was observed, in our serie, in the where no association between larger tumours
Table 1. Tumour Parameters and Nottingham Prognostic Groups

\begin{tabular}{lc}
\hline Characteristics & $\mathrm{N}(\%)$ \\
\hline Tumor size $(\mathrm{cm})$ & $41(41.8)$ \\
$\leq 3$ & $57(58.2)$ \\
$>3$ & \\
Lymph node status & $50(51.1)$ \\
$\mathrm{N}-$ & $48(48.9)$ \\
$\mathrm{N}+$ & \\
Tumor grade & $5(5.1)$ \\
I & $37(37.8)$ \\
II & $56(57.1)$ \\
III & \\
NPI prognostic groups & $5(5.1)$ \\
Good & $54(55.1)$ \\
Moderate & $39(39.8)$ \\
Poor &
\end{tabular}

displaying extensive lymph node invasion $(p=0.514)$. $50 \%$ of TNBC with sizes $\leq 3 \mathrm{~cm}$ displayed lymph node involvement whereas approximately $52.3 \%$ of TNBCs with sizes $>3 \mathrm{~cm}$ lacked axillary lymph node invasion.

\section{NPI and survival}

TNBC patients with NPI $>5.4$ are clearly separated from the good/moderate outcome curve in both OS $(p=0.001)$ (Figure 1A) and DFS at 5 years $(p<0.001)$ (Figure 1B), with an OS and DFS rates at 5 years,

Table 2. Association of Nottingham Prognostic Components to the Worst Outcome Group (NPI > 5.4)

\begin{tabular}{lccc}
\hline Characteristics & NPI $\leq 5.4(\%)$ & NPI $>5.4(\%)$ & $\mathrm{p}$ \\
\hline Tumor size $(\mathrm{cm})$ & & & 0,049 \\
$\quad \leq 3$ & 73.0 & 27.0 & \\
$>3$ & 45.5 & 54.5 & \\
Lymph node status & & & $<0.001$ \\
N - & 93.2 & 6.8 & \\
N + & 33.3 & 66.6 & \\
Tumor grade & & & 0,05 \\
I - II & 66.7 & 33.3 & \\
III & 44.7 & 55.3 & \\
\hline
\end{tabular}

Table 3. Univariate and Multivariate OS and DFS Analysis of NPI in TNBC

\begin{tabular}{lcccc}
\hline & Univariate $\mathrm{p}$ & Multivariate $\mathrm{p}$ & $\mathrm{HR}$ & $95 \% \mathrm{CI}$ \\
\hline OS & & & \\
Total 0.001 & 0.002 & & \\
$\mathrm{NPI} \leq 5.4$ & & 1 & - \\
$\mathrm{NPI}>5.4$ & & 2.598 & $1.423-4.744$ \\
$\mathrm{DFS}$ & & & \\
Total $<0.001$ & $<0.001$ & & \\
$\mathrm{NPI} \leq 5.4$ & & 1 & - \\
$\mathrm{NPI}>5.4$ & & 2.512 & $1.496-4.219$ \\
\hline
\end{tabular}




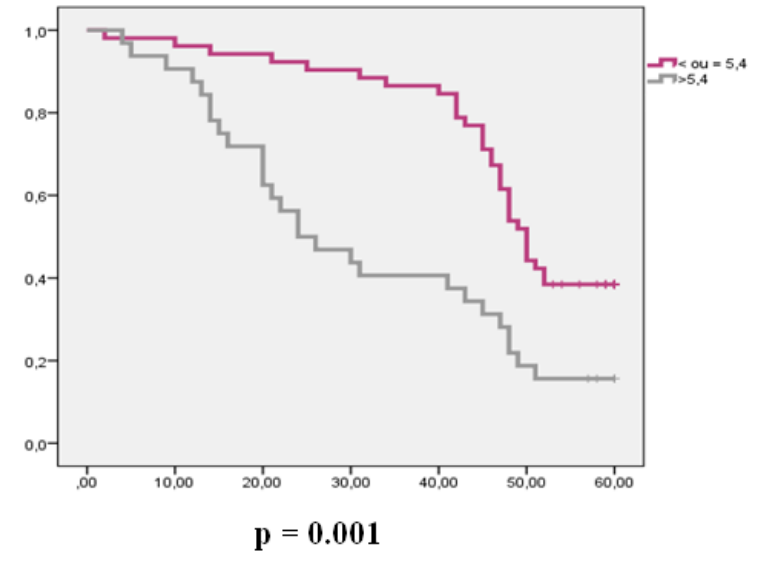

B

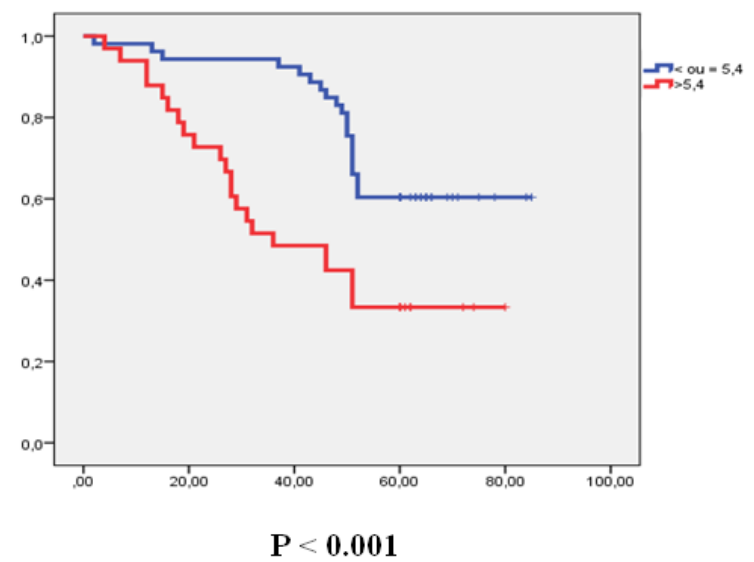

Figure 1. Kaplan Mieir Plots of Time to Mortality (A) and Time to Disease Progression (B) in TNBC Patients According to NPI ( $\leq 5.4$ vs. $>5.4)$

in the PPG, were 70 and $55.6 \%$ respectively, reinforcing not only the worse survival of the high-scored-NPI TNBC patients, but also suggesting the value of NPI as a predictor of survival in TNBC patients.

In multivariable analysis by Cox regression, high-scored NPI was significantly associated with OS (hazard ratio [HR]: $2.598,95 \%$ confidence interval [CI]: $1.423-4.744, \mathrm{p}=0.002)$ and DFS (HR: $2.512,95 \% \mathrm{CI}$ : $1.496-4.219, \mathrm{p}<0.001)($ Table 3$)$

\section{Discussion}

Patients with TNBC tend to have worse clinical outcomes partly as a result of lacking a therapeutic target. Chemotherapy is currently the mainstay of systemic medical treatment, although patients with triple negative disease, when considered as a group, have a worse outcome after chemotherapy than patients with breast cancers of other subtypes, a finding that reflects the intrinsically adverse prognosis associated with the disease. (Liedtke et al., 2008; Tan et al., 2008). Our results concerning tumour size (two thirds were $>3 \mathrm{~cm}$ ), greater histological grade $(56.6 \%)$ and high nodal involvement (48.7\%) are largely in accordance with literature data. (Derkaoui et al., 2016 ; Akasbai et al., 2011; Rais et al., 2011; Reddy et al., 2011; Zhao et al., 2009; Dent et al., 2009).

The poor prognosis of the TNBC was confirmed in our serie, the mean of NPI was 4.75 and none of our patient was scored as excellent prognostic. Similar findings were observed in a Spanish study analyzing the effectiveness of the Nottingham Prognostic Index in stratifying breast cancer patients of different subtypes with special emphasis in a 168 triple-negative breast cancer patient (Albergaria et al., 2011) and in the study conducted in Iran, including 233 early-stage breast cancer cases shows that $37.3 \%$ of the patients were classified in poor prognosis group (Rejali et al., 2015). Based on the current study, tumour size and grade variables are someway playing a compensatory score augmentation to NPI algorithm and considering that the majority of TNBC lesions are grade III (56.6\%), these largely studied prognostic factors are reliable to be used in the assessment of NPI in TNBC.

Interestingly, we didn't find an association between tumour size and lymph node status in TNBC $(p=0.514)$. This result contributes to some controversy concerning the existence or not of a relationship between these two prognostic factors in TNBC (Foulkes et al., 2008; Albergaria et al., 2011). According to our study and those published by Dent et al., (2007) and Spitale et al., (2009), TNBC do not seem to obey the "size-node" rule.

The present study confirms reports that High - scored NPI (>5.4) independently predict OS and DFS in TNBC patients. Our results agreed with the Spanish study published by Albergaria group (Albergaria et al., 2011), which showed that increasing NPI is related to a poor outcomes and short survivorship, suggesting the reliability of NPI as a tool to be reproducibly used in TNBC tumours. Furthermore, based on a retrospective study of 1661 retrospective study of 1661 breast cancer patients, the value of NPI as a practicable prognostic tool was confirmed for brest cancer, even for the TNBC subtype (Wen et al., 2015).

Some studies indicate a possible improvement of the categorization of patients by incorporating the ER, PR, and HER2 into NPI. An Improving NPI (iNPI) was developed and studied: it increases 5-year DFS in 1,927 patients with primary operable breast cancer. So, according to this study published in JCO in 2010 (Van belle et al., 2010), the iNPI can be considered as a clinically useful tool for stratification of patients with breast cancer. Another score (Extended NPI) was developed using suitable statistical methodology to extract the full information from standard clinical data. This extended version of NPI can serve as a benchmark to assess the added value of new information and leads to an important increase in its prognostic ability. (Winzer et al., 2016)

It is now recognized that the biological characteristics of $\mathrm{BC}$ are important for clinical management and addition of biological markers to the NPI can significantly improve risk stratification of BC patients (Rakha et al., 2014). A new score (NPI plus) which conceptually evolved to modernize the historical NPI by applying the prognostic methods used in the NPI, which are based on well-established 
powerful clinicopathological variables, following $\mathrm{BC}$ molecular class assignment, was analyzed. The NPI plus $(\mathrm{NPI}+)$ was developed using the following biomarkers: Estrogen Receptor (ER), Progesterone Receptor (PgR), cytokeratin (CK) 5/6, CK7/8 (using the anti-cytokeratin CAM5.2 clone), epidermal growth factor receptor (EGFR; HER1), c-erbB2 (HER2), c-erbB3 (HER3), c-erbB4 (HER4), p53 and Mucin 1 (Green AR et al., 2014). The molecular classes identified based on the combination of these 10 biomarkers are three luminal classes (Luminal $\mathrm{A}, \mathrm{N}$ and $\mathrm{B}$ ), two basal classes (Basal - p53 altered and Basal - p53 normal) and two HER2+ classes (HER2+l ER+ and HER2+/ER-) (Soria et al., 2013). The study conducted by Green et al., (2016), demonstrated the reproducibility of $\mathrm{NPI}+$ and confirmed its prognostic value in an independent cohort of 885 primary BC.

Our study seems to be the first study to examine the relationships between NPI and TNBC survival outcomes in Moroccan patients, to our knowledge. However, there were some potential limitations in our study, represented by: the retrospective nature of the study design and its relatively small sample size.

Our study established a relationship between NPI and TNBC outcomes. Once validated by prospective study, these results should be taken into account in the medical management by using intensified treatments in patients with high-scored NPI.

\section{References}

Akasbai Y, Bennis S, Abbass F, et al (2011). Clinicopathological, therapeutic and prognostic features of the triple-negative tumors in Moroccan breast cancer patients (experience of Hassan II university hospital in Fez). BMC Res Notes, 2011, 500 .

Albergaria A, Rcardo S, Milanezi F, et al (2011). Nottingham prognostic index in triple-negative breast cancer : a reliable prognostic tool?. BMC Cancer, 2011, 299.

Balmey RW, Ellis IO, Pinder SE, et al (2007). Survival of invasive breast cancer according to the Nottingham Prognostic Index in cases diagnosed in 1990-1999. Eur $J$ Cancer, 43, 1548-55.

Dent R, Trudeau M, Pritchard KI, et al (2007). Triple-negative breast cancer : clinical features and patterns or reccurence. Clin Cancer Res, 13, 4429-34

Dent R, Hanna WM, Trudeau M, et al (2009). Pattern of metastatic spread in triple-negative breast cancer. Breast Cancer Res Traet, 115, 423-8.

Derkaoui T, Bakkach J, Mansouri M, et al (2016). Triple negative breast cancer in North of Morocco: clinicopathologic and prognostic features. BMC Womens Health, 16, 68.

D'Eredita G, Giardina C, Martellotta, et al (2001). Prognostic factors in breast cancer: the predictive value of the Nottingham Prognostic Index in patients with a long-term follow-up that were treated in a single institution. Eur $J$ Cancer, 375, 591-6.

Fong Y, Evans J, Brook D, et al (2015). The Nottingham Prognostic Index : five- and ten-year data for all-cause Survival within a Screened Population. Ana R Coll Surg Engl, 97, 137-9.

Foulkes WD, Grainge MJ, Rakha EA, et al (2008). Tumor size is an unreliable predictor of prognosis in basal-like breast cancers and does not correlate closely with lymph node status. Breast Cancer Res Treat, 117, 199-204.
Fulford LG, Reis-Filho JS, Ryder K, et al (2007). Basal-like grade III invasive ductal carcinoma of the breast: patterns of metastasis and long-term survival. Breast Cancer Res, 9, R4.

Galea MH, Blamey RW, Elston CE, et al (1992). The Nottingham prognostic index in primary breast cancer. Breast Cancer Res Treat, 22, 207-19.

Green AR, Powe DG, Rakha EA, et al (2013). Identification of key clinical phenotypes of breast cancer using a reduced panel of protein biomarkers. Br J Cancer, 109, 1886-94.

Green AR, Soria D, Stephen J, et al (2016). Nottingham Prognostic Index Plus: Validation of a clinical decision making tool in breast cancer in an independent series. $J$ Pathol Clin Res, 2, 32-40.

Haybittle JL, Blamey RW, Elston CW, et al (1982). A prognostic index in primary breast cancer. Br J Cancer, 45, 361-6.

Hicks DG, Short SM, Prescott NL, et al (2006). Breast cancers with brain metastases are more likely to be estrogen receptor negative, express the basal cytokeratin CK5/6, and overexpress HER2 or EGFR. Am J Surg Pathol, 30, 1097-1104.

Jeanny K, Keun-Young E, Tae RK, et al (2017). A prognostic model for patients with triple-negative breast cancer: Importance of the modified nottingham prognostic index and age. J Breast Cancer, 20, 65-73

Liedtke C, Mazouni C, Hess KR, et al (2008). Response to neoadjuvant therapy and long-term survival in patients with triple-negative breast cancer. J Clin Oncol, 26, 1275-81.

Perou CM, Sorlie T, Elisen MB, et al (2000). Molecular portraits of human breast tumours. Nature, 406, 747-2.

Rais G, Raissouni S, Aitelhaj M, et al (2012). Triple negative breast cancer in Moroccan women : clinicopathological and therapeutic study at tha National Institute of Oncology. BMC Womens Health, 12, 35.

Rakha EA, Soria D, Green AR, et al (2014). Nottingham Prognostic Index Plus (NPI+): a modern clinical decision making tool in breast cancer. Br J Cancer, 110, 1688-97.

Reddy KB (2011). Triple-negative breast cancers: an updated review on treatment options. Curr Oncol, 18, 173-9.

Rejali M, Tazhibi M, Mokarian F, et al (2015). The performance of the Nottingham prognosis index and the adjuvant online decision making tool for prognosis in early-stage breast cancer patients. Int J Prev Med, 6, 93.

Soria D, Garibaldi JM, Green AR, et al (2013). A quantifierbased fuzzy classification system for breast cancer patients. Artif Intell Med, 58, 175-84.

Spitale A, Mazzola P, Soldini D, et al (2009). Breast cancer classification according to immunohistochemical markers: clinicopathologic features and short-term survival analysis in a population-based study from the South of Switzerland. Ann Oncol, 20, 628-35.

Tan DS, Marchío C, Jones RL, et al (2008). Triple negative breast cancer: molecular profiling and prognostic impact in adjuvant anthracycline-treated patients. Breast Cancer Res Treat, 111, 27-44.

Van Belle V, Van Calster B, Brouckaert O, et al (2010). Qualitative assessment of the progesterone receptor and HER2 improves the Nottingham Prognostic Index up to 5 years after breast cancer diagnosis. $J$ Clin Oncol, 28, 4129-34.

Wen J, Ye F, Li S, et al (2015). The practicability of a novel prognostic index (PI) model and comparison with Nottingham prognostic index (NPI) in stage I-III breast cancer patients undergoingsurgical treatment. PLoS One, 10, e0143537.

William D, Foulkers MB, Ian E, et al (2010). Triple-negative breast cancer. N Engl J Med, 363, 1938.48.

Winzer KJ, Buchholz A, Schumacher M, et al (2016). Improving 
the prognostic ability through better use of standard clinical data - the Nottingham prognostic index as an example. PLoS One, 11, e0149977.

Zhao J, Liu H, Wang M, et al (2009). Characteristics and prognosis for molecular breast cancer subtypes in Chinese women. J Surg Oncol, 100, 89-94

\section{(ब) $(1) \Theta$}

This work is licensed under a Creative Commons AttributionNon Commercial 4.0 International License. 